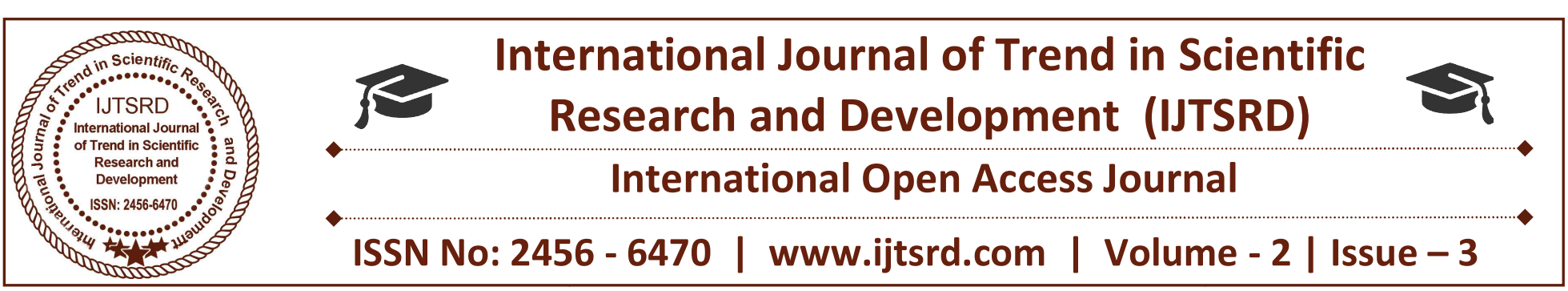

\title{
Hot Disk Pick and Place Robot
}

\author{
Rizwaan Areef Shaikh, Ajinkya Ramesh Vadaje, Tejaswi Uttam Zagade \\ UG Student, Department of Mechanical Engineering, \\ Shatabdi Institute of Engineering \& Research, Nashik, Maharashtra, India
}

\begin{abstract}
The Project is aimed to provide an automation in handling of different type of hot discs prior to forming operation.

In Present situation, hot discs (approx. at $750^{\circ} \mathrm{c}$ ) are guided to operator by conveyor from the furnace. Operator pics the disc with the help of tounge and places it on forming setup. The process is slightly time consuming, due to that the temperature of disc decreases and that results in decrease in forming quality. In addition to that, as the weight of disc is quite high (22 to $35 \mathrm{~kg} /$ disc), the process was taking large amount of human efforts. To address all these issues, the team decided to Design a pick and place type robot in the work area.
\end{abstract}

The main objective of our project is to provide a pick and place robot which will handle all 12 types of discs with one End effector and also it must take minimum time to move the hot disc from conveyor to the forming setup to save time and increase productivity.

Keyword: Material handling robot, Robotic Arm, PLC

\section{INTRODUCTION}

Robotics deals with the design of robots their manufacture and applications. Robotics gained more importance in the modern era since it require less cost to operate than a human labour to do the same task, also once programmed robot will perform better than an experienced human labour. Now a days industry is turning towards computer based monitoring of tasks mainly due to the need for the increased productivity and delivary of the final products with maximum quality. Due to the inflexibility and generally high cost of hard computerization systems lead to the use of industrial robots.

Material handling can be defined as an integrated system involving such activities as moving, handling, storing and controlling of materials by means of gravity, manual effort or power activated machinery. Moving materials utilize time and space. Any movement of materials requires that the size, shape, weight and condition of the material, as well as the path and frequency of the move be analyzed

\subsection{Problem Definition}

hot discs (approx. at 900c) are guided to operator by conveyor from the furnace. Operator pics the disc with the help of tounge and places it on forming setup. The process is slightly time consuming, due to that the temperature of disc decreases and that results in decrease in forming quality so to counter that plate need to put in funace again and that was affecting production targets. In addition to that, as the weight of disc is quite high (22 to $35 \mathrm{~kg} / \mathrm{disc}$ ), the process was causing backbone problems for operator and also there is higher chances of an accident. Process is not satisfying MNC norms for safety of operator.

The easiest way to prepare your document is to use this document as a template and simply type your text into it.

\subsection{Objective}

1. Our main objective is to make sure Robot will move hot disc from conveyor to forming setup in less than $13 \mathrm{sec}$. 
2. To eliminate human interface.

3. To make the Robot which is Reliable in any hostile Environment.

4. To improve productivity.

5. To Improve Quality of product.

\subsection{Scope}

1. In future we can improve (lower) cycle time as well as positional accuracy by using Servomotor for all three axis.

2. Clamping method can also be improved by providing large clamping area.

\section{Methodology}

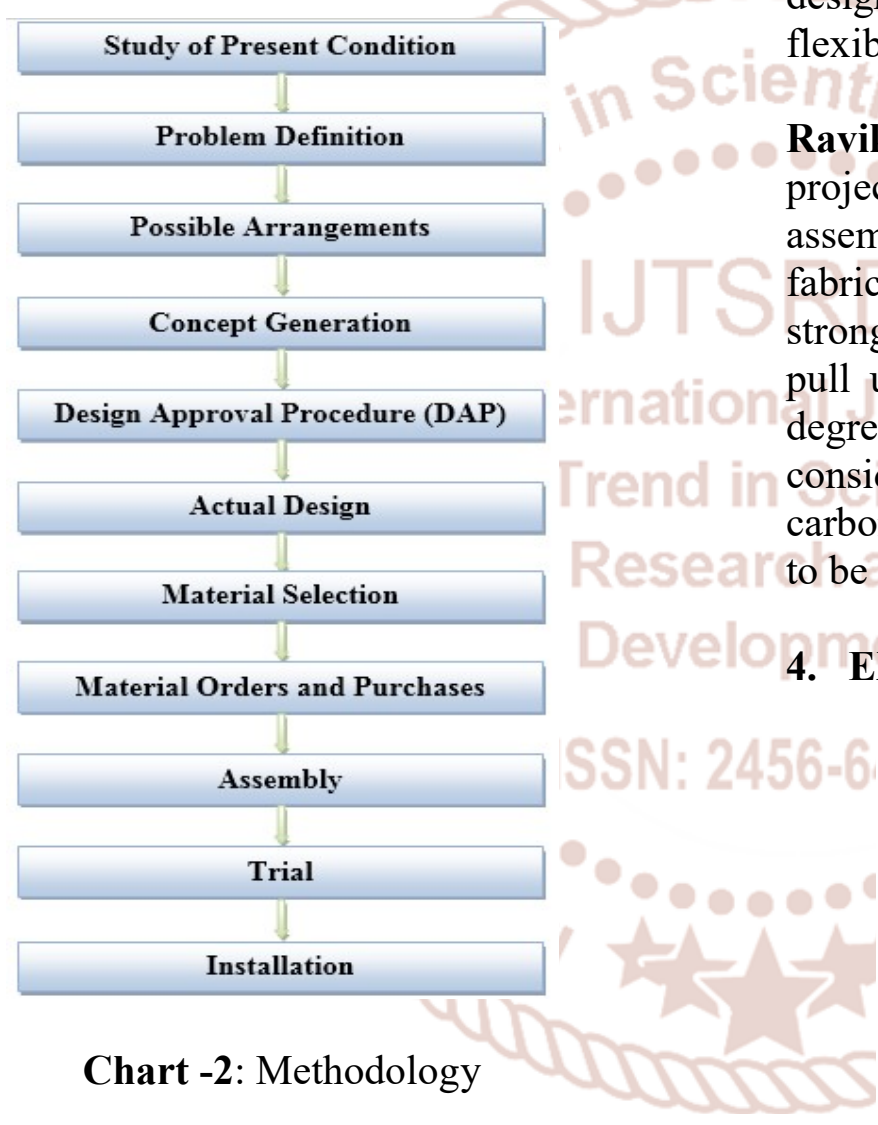

\section{LITERATURE SURVEY}

\section{Modi Akshaykumar Pravinkumar D. Patel [1]: \\ Mahendrakumar,}

As per this project, the limitations of human work should be solved. The human works have many limitations likewise, laziness, less accuracy, time limits, less productivity, etc. In the industrial world, automation is one of the essential elements for development. It helps to reduce the need for humans and increase efficiency and productivity. The field of automation occupies large areas, mostly in industrial manufacturing. A robotic arm is a type of mechanical arm, usually programmable, with similar functions to a human arm. The links of such a manipulator are connected by joints allowing either rotational motion or linear displacement. Used for assembly operations, diecasting, gas welding, arc welding and spray painting,etc.

Mir Sajjad Hussain Talpur, Murtaza Hussain Shaikh [2]: As per this project, aim is to design and implement a PLC based on reliable and high performance robotic system for food / biscuit manufacturing line.we are referring a robot, which is capable of picking unbaked biscuits tray and places them into furnace and then after baking it picks the biscuits tray from thefurnace. A special gripper is designed to pick and place the biscuits tray with flexibility.

Ravikumar Mourya , Amit Shelke[3]:In this project, mechanical construction is to build and assemble the robot body. The most suitabl material to fabricate the structure of the arm has to be light and strong. Otherwise, the servo motor will not be able to pull up the arm and to perform the desired turning degree. among the four materials considered(aluminum, Perspex, plastic polymer and carbon fiber), the aluminum is the most ideal material to be chosen as fabrication material

\section{EXPERIMENTAL WORK}

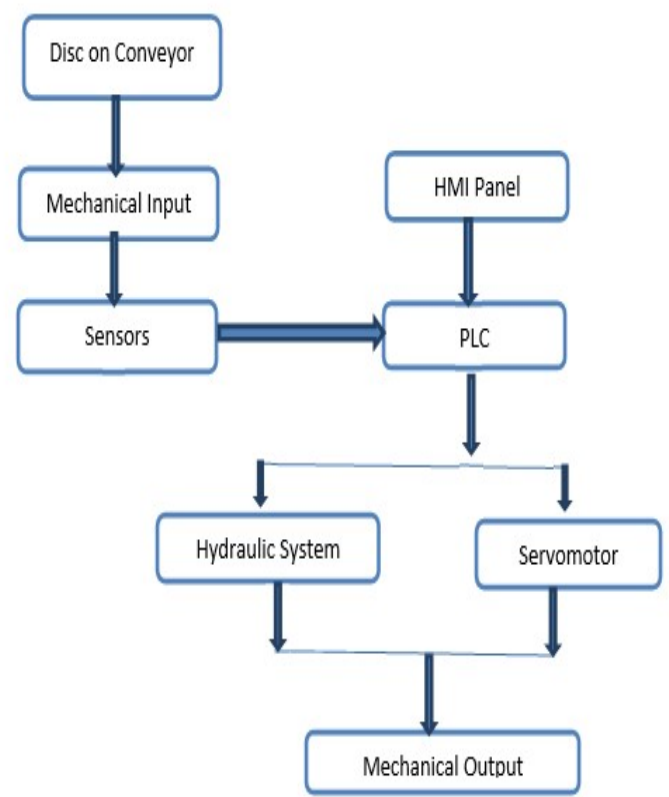

Chart -2: Process flow diagram

\subsection{Working}

Hot disc from furnace is passed towards robot with the help of conveyor. As the disc touches end effector 
, it gets actuated and holds the disc firmly. Robot have many positional sensors, hence when the input signal comes from PLC, hydraulic system and servomotor gets actuated, and produces desired output i.e hot disc gets placed accurately on loacater pin of forming setup.

\section{CONCLUSION}

In this way, we conclude that this hot disc pick and place robot can be used to move the hot disc from conveyor to forming setup accurately and it can work in any hostile environment.

\section{ACKNOWLEDGEMENT}

I express my sincere gratitude to my guide Prof. $\mathbf{H}$. S.Shinde for his technical support which made this Project stage - I possible. His constant encouragement, suggestions and ideas have been in valuable to this work. I immensely appreciate the time he devoted reviewing my writing and vastly improving my technical writing skills. His thoroughness, discipline and work ethic are laudable and worthy of emulation. I am grateful to them for providing their valuable time without that this dissertation is not possible.

I would also like to thank Prof. P. G. Vispute, Principal for reviewing my work. I am also grateful to Prof. S.D. Ratnakar, Head of Department of Mechanical Engineering for constant support and for providing me with all possible facilities in the college. I would also like to thank the entire Faculty Members of Mechanical Engineering Department for all their

\section{REFERENCES}

1. Modi Akshaykumar Mahendrakumar, Pravinkumar D. Patel, Dodia Bhagirathkumar Varjangbhai, Moradiya Chiragkumar Khodabhai, Bhavesh Prajapati "design and development of pick and place robotics arm Using plc-scada" 1 st International Conference on Current Research in Engineering. Date: $13^{\text {th }}$ April 2017 Page No.: 1- 3

2. Mir Sajjad Hussain Talpur, Murtaza Hussain Shaikh "Automation of Mobile Pick and Place Robotic System for Small food Industry" Patent Application Publication Date:28 ${ }^{\text {th }}$ June2007 Page No.: 1 - 7

3. Ravikumar Mourya , Amit Shelke, Sourabh Satpute, Sushant Kakade , ManojBotre"Design and Implementation of Pick and Place Robotic Arm" International Journal of Recent Research in Civil and Mechanical Engineering Date: Sept 2015 Page No.: 2 - 3 